\title{
Hipertensión Pulmonar Crónica Tromboembólica Tromboendarterectomía Pulmonar
}

\author{
Francisco José Ayala ${ }^{1}$, Juan Espinoza ${ }^{1}$, Luigi Lagazzi ${ }^{2}$, Mauricio González ${ }^{3}$, Fernanda Anich $^{a}$, Gabriela Soto $^{a}$, José Robles ${ }^{4}$, \\ Francisco Araneda ${ }^{5}$, Felipe Quintas ${ }^{6}$, Marcelo Poblete ${ }^{6}$. \\ 1. Departamento Cardiovascular, Hospital Clínico Universidad de Chile, Santiago, Chile. \\ 2. Cardiothoracic Surgeon, Chief Mechanical Circulatory Support, Hunter Holmes McGuire VAMC, Richmond, VA. Faculty Virginia Comonwealth. \\ University (VCU) Cardiothoracic Surgery Program. \\ 3. Departamento de Anestesiología, Hospital Clínico Universidad de Chile, Santiago, Chile. \\ a. Escuela de Medicina, Facultad de Medicina, Universidad de Chile, Santiago, Chile. \\ 4. Departamento de Cirugía, Hospital Clínico Universidad de Chile, Santiago de Chile. \\ 5. Departamento Nutrición y Diabetes, Escuela de Medicina, Pontificia Universidad Católica de Chile, Santiago, Chile. \\ 6. Departamento de Imagenología, Hospital Clínico Universidad de Chile, Santiago, Chile.
}

Fuente de financiamiento: Los autores no recibieron patrocinio para llevar a cabo este artículo.

Conflicto de intereses: Los autores declaran no tener conflicto de intereses.

\section{Resumen:}

A nivel mundial, la tercera causa de muerte por causa cardiovascular es el Tromboembolismo Pulmonar (TEP), después del Infarto agudo de Miocardio y el Accidente cerebrovascular, con una incidencia anual estimada de 40 casos por cada 100.000 habitantes.

Se comunica el caso clínico de un paciente de 44 años con diagnóstico de TEP recurrente con Hipertensión pulmonar tromboembólica crónica (CTPH) que fue some- tido a tromboendarterectomía pulmonar bilateral (PTE) bajo paro circulatorio con hipotermia profunda. Se informa de los resultados de los medios de diagnóstico y del tratamiento quirúrgico mediante la tromboendarterectomía. Se discute el tema en el contexto de la experiencia internacional y nacional.

Palabras clave: Embolía Pulmonar, Hipertensión Pulmonar Crónica, Tromboembolismo, Tromboendarterectomía.

\section{Correspondencia:}

Dr. Francisco José Ayala R.

ayalafranciscojose@gmail.com, frayala@hcuch.cl. 


\section{Caso Clínico:}

Presentamos el caso de un paciente hombre de 44 años con antecedente de tabaquismo crónico suspendido hace 25 años (IPA 1). Sin antecedentes de uso de drogas y fármacos. Dentro de la historia familiar destaca padre fallecido por TEP a los 49 años y una hermana con trombofilia primaria por déficit de proteína C. Consultó por cuadro de 6 meses de evolución de disnea de esfuerzo progresiva con deterioro de CF I a CF III (NYHA), edema de extremidades inferiores y disnea paroxística nocturna. Una semana previo al ingreso, refirió episodio de sensación febril que se interpretó como cuadro respiratorio tratado con antibióticos, sin mejoría clínica. El ecocardiograma (ECO) transtorácico ambulatorio evidenció dilatación severa de cavidades derechas, sobrecarga de ventrículo derecho (VD), Insuficiencia Tricuspídea severa y Presión Sistólica de Arteria Pulmonar (PSAP) de $85 \mathrm{mmHg}$.

El ECO de control mostró hipertensión pulmonar (HTP) severa con presión sistólica de arteria pulmonar (PSAP) de $141 \mathrm{mmHg}$, VD dilatado (56 x $78 \mathrm{~mm}$ ), hipertrofia de pared libre y signos de disfunción sistólica moderada, severa insuficiencia Tricuspídea, insuficiencia moderada de válvula pulmonar, dilatación de aurícula derecha (AD), septum interventricular desplazado a la izquierda con movimiento paradojal y fracción de eyección del Ventrículo Izquierdo (FEVI) 62\%. El angio TAC de tórax (ATT) documentó TEP bilateral de ramas principales con extensión segmentaria, elementos de cronicidad y signos de sobrecarga VD con HTP secundaria.

Presentó reacción adversa a tratamiento anticoagulante con heparina sódica no fraccionada, siendo tratado finalmente con Fondaparinux. La evolución fue tórpida con aumento de disnea, alto requerimiento de oxígeno y episodio sincopal. La trombolisis con t-PA se asoció a una mejoría ventilatoria parcial. Con el manejo médico con sildenafil y espironolactona, y con la desensibilización de heparina a dosis crecientes, se logró estabilización de su condición general con disminución de la disnea.

El estudio de Trombofilia resultó negativo para AT III, Factor V de Leyden, mutación del gen de la protrombina, y para la presencia de anticuerpos anticardiolipina. Hubo elevación discreta de la homocisteína. El ECO Doppler venoso de extremidades inferiores mostró signos de trombosis venosa profunda y presencia de trombo flotante en vena femoral común, superficial. Por el riesgo de recurrencia de TEP se implantó un filtro en vena cava inferior.

El ECO control mostró persistencia de HTP severa y signos de falla derecha. El ATT evidenció defectos de llene

\begin{tabular}{|c|c|}
\hline Parámetro & Valor \\
\hline Frecuencia cardíaca & $86 \mathrm{lpm}$ \\
\hline Gasto cardíaco (termodilución) & $5,4 \mathrm{~L} / \mathrm{min}$ \\
\hline Gasto cardíaco (Fick) & $3,46 \mathrm{~L} / \mathrm{min}$ \\
\hline Índice cardíaco & $1,95 \mathrm{~L} / \mathrm{min} / \mathrm{m}^{2}$ \\
\hline Arteria pulmonar & $67 / 24(40) \mathrm{mmHg}$ \\
\hline Capilar pulmonar & $11 \mathrm{mmHg}$ \\
\hline Resistencia pulmonar & 786 dinas $/ \mathrm{s} / \mathrm{cm}^{-5} / 9,8 \cup$ Wood \\
\hline Qp/Qs & $1,0 / 1,0$ \\
\hline Test de Adenosina & 250 UI/L Discreta respuesta \\
\hline Test de Prostaglandinas & Resultados discretos y mala tolerancia \\
\hline
\end{tabular}

periféricos por presencia de trombos antiguos, patrón de atenuación en mosaico en ambos pulmones compatible con TEP crónico, signos de HTP y sobrecarga de VD (Figura 1). La arteriografía pulmonar y el cateterismo cardíaco derecho (CCD) demostraron TEP crónico con severo compromiso del lóbulo inferior izquierdo y pulmón derecho e HTP severa (Tabla 1). El cintigrama V/Q evidenció múltiples defectos de perfusión con ventilación conservada y alta probabilidad de TEP; las fracciones de eyección de VD y de VI fueron $40 \%$ y $41 \%$, respectivamente.

El paciente logró mejoría parcial en test de marcha desde $37 \%$, a $62 \%$ del valor teórico.

Fue hospitalizado en forma anticipada tras presentar 2 nuevos episodios sincopales que se relacionaron con accesos de tos. El cateterismo cardíaco derecho (CCD) y la angiografía pulmonar demostraron TEP crónico recurrente con severo compromiso del lóbulo medio e inferior derecho y del lóbulo inferior izquierdo con recanalización parcial, HTP severa y resistencias vasculares pulmonares (RVP) en rangos compatibles con la indicación de cirugía (Tabla 2).

Se efectuó una esternotomía media vertical, paro circulatorio en hipotermia profunda $\left(20^{\circ} \mathrm{C}\right)$, pericardiotomía en T invertida, canulación arterial ascendente y bicaval, con cardioplejia anterógrada, vent aórtico y de AP con bypass cardiopulmonar. Se procedió a una tromboendarterectomía bilateral en vasos pulmonares segmentarios y 
Tabla 2: Cateterismo cardíaco derecho (Segundo estudio) post Trombolisis Pre quirúrgico (Tromboendarterectomía Pulmonar).

\begin{tabular}{|l|c|}
\hline Parámetro & Valor \\
\hline Frecuencia cardíaca & $69 \mathrm{lpm}$ \\
\hline Gasto cardíaco (termodilución) & $4,4 \mathrm{~L} / \mathrm{min}$ \\
\hline Índice cardíaco & $2,53 \mathrm{~L} / \mathrm{min} / \mathrm{m}^{2}$ \\
\hline AD & $(10) \mathrm{V}=13$ \\
\hline VD & $90 / 1-12$ \\
\hline Presión AP & $83 / 25(45) \mathrm{mmHg}$ \\
\hline Resistencia pulmonar total & $863,6 \mathrm{dinas} / \mathrm{s} / \mathrm{cm}^{-5}$ \\
\hline $\begin{array}{l}\text { AD: Aurícula Derecha } \\
\text { VD: Ventrículo Derecho } \\
\text { AP: Arteria pulmonar }\end{array}$ & \\
\hline
\end{tabular}

subsegmentarios, mediante arteriotomía pulmonar bilateral, disección y endarterectomía de los planos hasta nivel subsegmentario en ambos pulmones. Se extrajo abundante material trombótico crónico adherido a las paredes de los vasos pulmonares segmentarios y subsegmentarios, mayor en pulmón derecho (Figura 2). El ETE postoperatorio mostró mejoría de la función del VD, insuficiencia tricuspídea leve, disminución de PAPS de 85 a $25 \mathrm{mmHg}$ y RVP baja. Egresó de pabellón con apoyo de dopamina en dosis bajas + pacing auricular.

La evolución fue favorable con corrección de parámetros imagenológicos, hemodinámicos (RVP bajas, PAPM de $20 \mathrm{mmHg}$ ) y respiratorios. Presentó edema pulmonar por reperfusión, siendo extubado a las 48 horas. El ECO control documentó reducción de PAPS (32 mmHg), cinética del VD conservada, movimiento paradojal del septum interventricular, función del ventrículo izquierdo (VI) conservada. Fue dado de alta a los 12 días postoperatorio con test de marcha alcanzando $75 \%$ del valor teórico.

El ATT control no evidenció signos de recurrencias de TEP, cámaras cardíacas de tamaño conservado, relación VD/VI $<1$, tronco de AP $29 \mathrm{~mm}$ de diámetro (Figura 1). El ECO mostró PAPS normal $(30 \mathrm{mmHg})$, cavidades derechas levemente dilatadas, insuficiencia tricuspídea leve, función sistólica levemente disminuida del VD, VI de dimensiones y función sistólica normales, septum paradojal, y disfunción diastólica.

Actualmente, el paciente se encuentra asintomático, cumple tratamiento anticoagulante oral a permanencia con seguimiento hasta dos años posterior a la cirugía, con buena calidad de vida (Capacidad Funcional I).
Figura 1.- Tomografía de Tórax con contraste preoperatoria (columna izquierda) y post Tromboendarterectomía (columna derecha), ambas en fase angiográfica.
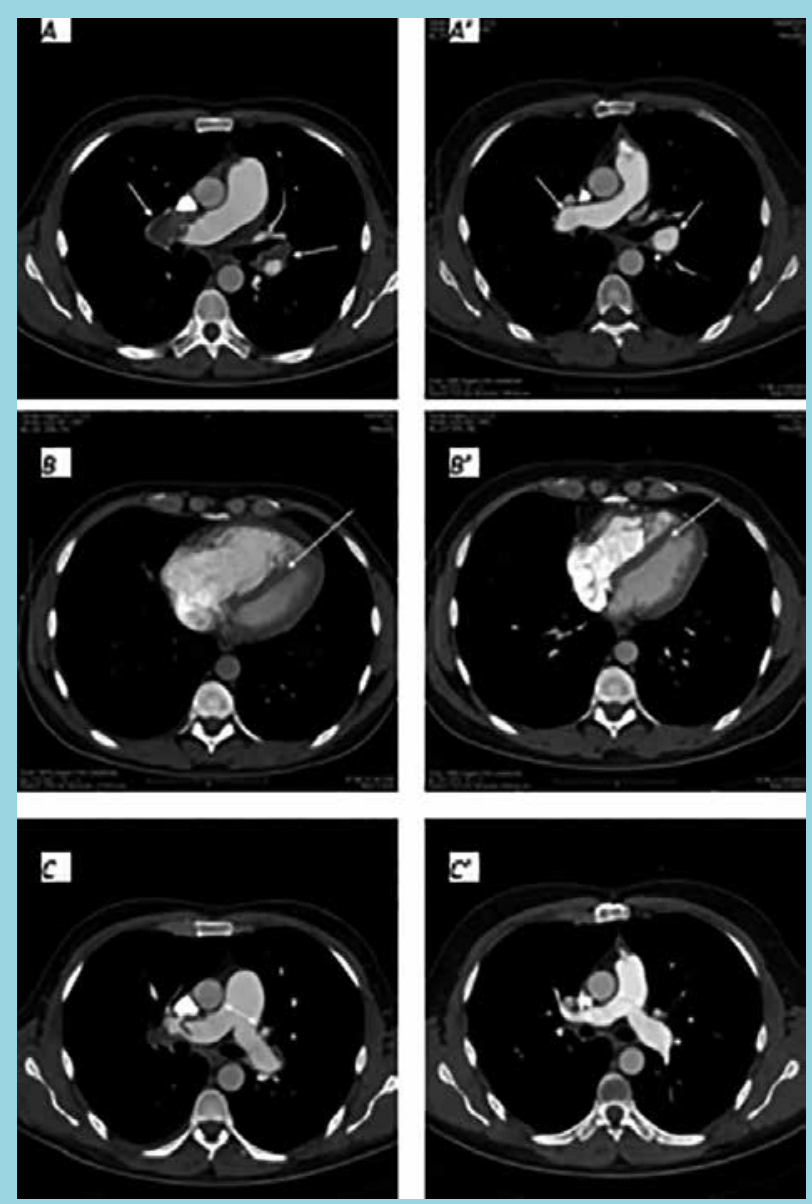

Imagen A: Presencia de trombo que ocluye la totalidad de la arteria pulmonar derecha (flecha blanca). En imagen A', post Tromboendarterectomía, se observa la arteria pulmonar derecha permeable (flecha blanca).

Imagen B: Muestra abombamiento del septum interventricular con convexidad izquierda, signo de sobrecarga de cavidades derechas. En imagen B', se observa el septum interventricular de morfología conservada, lo que traduce regresión de la sobrecarga.

Imagen C: Dilatación patológica del tronco de la arteria pulmonar (flecha blanca). En imagen $C$ ', se observa el tronco de la arteria pulmonar de diámetro normal (flecha blanca).

\section{Discusión:}

A nivel mundial, la tercera causa de muerte por causa cardiovascular es el TEP, con incidencia anual estimada de 40 casos por cada 100.000 habitantes ${ }^{1}$.

De los pacientes que sufren TEP, la mitad normaliza su función pulmonar, hemodinamia, intercambio gaseoso y tienen buena tolerancia al ejercicio. Estudios han evidenciado que posterior a una embolia pulmonar, $45-52 \%$ de los pacientes desarrollan insuficiencia cardíaca (IC) 
Figura 2: Hallazgos intraoperatorios de Tromboendarterectomía Pulmonar.
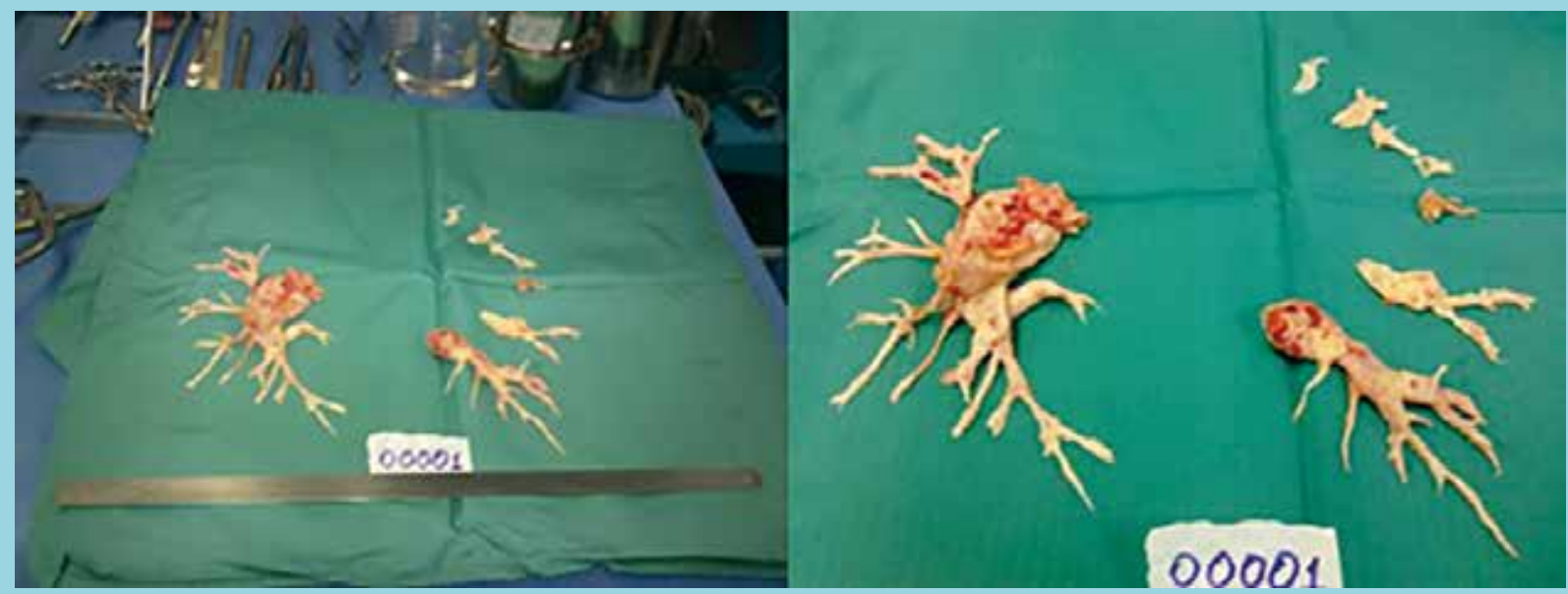

Abundante material trombótico crónico adherido a las paredes de los vasos pulmonares segmentarios y subsegmentarios.

derecha de al menos grado II (NYHA) y entre el 0,56-3\% presentará $\mathrm{CTPH}^{2,3}$. La CTPH es definida como Presión de Arteria Pulmonar Media (PAPM) mayor a $25 \mathrm{mmHg}$ que persiste 6 meses después del diagnóstico de TEP o posterior a 3 meses de tratamiento con anticoagulación en rangos terapéuticos ${ }^{5}$.

La CTPH es subdiagnosticada, con una incidencia esperada de 1,6 a 35,2 casos por millón de habitantes, lo que está por sobre algunos valores reportados ${ }^{1}$. Es de difícil diagnóstico por presentar síntomas inespecíficos. Se puede confundir con episodios de TEP, enfermedad coronaria o EPOC, especialmente en pacientes en los que no hay antecedentes de tromboembolismo venoso agudo ${ }^{1}$. La presentación clínica es similar a cualquier otra forma de HTP. El inicio es subclínico, siendo la disnea de esfuerzo y la intolerancia al ejercicio los síntomas más comunes. Otras manifestaciones clínicas incluyen: tos, hemoptisis, síncope, mareos y dolor torácico. Los pacientes con disnea, intolerancia al esfuerzo e IC derecha, presentan mayor mortalidad ${ }^{4,6}$. Los signos son aumento de presión venosa yugular, desdoblamiento de S2 con P2 acentuado, galope $\mathrm{S} 4$, soplo de regurgitación tricuspídea $\mathrm{y}$ en etapas avanzadas hepatomegalia, ascitis y edema periférico 5 .

No está claro por qué algunos pacientes con TEP desarrollan CTPH y otros no. Estudios sugieren que la causa sería multifactorial, destacando entre ellos las vasculopatías, esplenectomía previa, shunts ventrículo-atriales y estado procoagulante subyacente ${ }^{7}$. Causas menos frecuentes son deficiencia de antitrombina III, proteína $\mathrm{C}$ o proteína $\mathrm{S}^{8}$.

La causa de la HTP en la condición de CTPH aparentemente no sería sólo producto de embolias recurrentes, ni formación de trombos in situ, sino además consecuencia de cambios en la resistencia de pequeños vasos en la circulación pulmonar periférica, remodelamiento con desarrollo de bandas, redes de colágeno, fibroblastos e inflamación en la capa íntima 9 .

La utilidad de los estudios de imágenes para el diagnóstico y tratamiento dependerá de la ubicación del trombo y del grado de sospecha clínica.

La Radiografía de tórax puede ser normal o mostrar signos de HTP como hilios pulmonares prominentes, crecimiento de AD y VD e infarto pulmonar ${ }^{5}$. El ECO transtorácico es fundamental para evaluar la función del VD, crecimiento de cavidades derechas, función de la válvula tricúspide, PSAP y presencia de derrame pericárdico. No permite diferenciar distintas etiologías que pudiesen haber originado la $\mathrm{CTPH}^{10}$.

El AT pulmonar tiene alta sensibilidad. Aporta información de extensión de patología en el parénquima pulmonar, anatomía y tamaño de vasculatura pulmonar, localización de vasos colaterales y crecimiento de cavidades derechas ${ }^{10}$. El CCD es el gold standard. Evalúa la severidad de la HTP, la disfunción cardíaca en reposo, define los candidatos a cirugía y predice la mortalidad perioperatoria. Aporta análisis del grado de enfermedad de vaso pequeño, ayudando a predecir el resultado quirúrgico ${ }^{10}$. La angiografía pulmonar muestra organización y recanalización de la CTPH. Es de utilidad para predecir los 
resultados de la cirugía y el éxito de ésta ${ }^{10}$. El uso de resonancia magnética y ATT se está expandiendo. Permiten evidenciar cambios vasculares de la CTPH, el diagnóstico de TEP agudo e insuficiencia ventricular derecha ${ }^{10}$. La terapia definitiva y potencialmente curativa del CTPH para algunos casos sería la PTE ${ }^{11}$. Los principales abordajes quirúrgicos son la endarterectomía bilateral y completa, recomendada por la Universidad de California de San Diego ${ }^{12}$. La Clasificación de Jamieson del tipo de trombo y su localización ${ }^{13}$ también constituyen factores pronósticos. La experiencia del equipo quirúrgico es el factor más relevante para disminuir los riesgos y mortalidad relacionada a este procedimiento ${ }^{14}$.

El estudio de los pacientes con CTPH para evaluar si son candidatos a esta cirugía es trascendental, ya que esta condición patológica presenta muy mala sobrevida a corto plazo. En esta evaluación, los objetivos son establecer si la PTE es factible y luego determinar si la cirugía es de beneficio para el paciente. Una RVP $<1.200$ dinas $/ \mathrm{s} / \mathrm{cm}^{5}$ y ausencia de comorbilidades son factores de pronóstico favorable 4 .

En la literatura nacional se comunica el primer caso exitoso de PTE como tratamiento quirúrgico de TEP crónico bajo paro circulatorio con hipotermia profunda. El paciente descrito por Zalaquett y cols ${ }^{15}$ presentó características similares al caso reportado, sin respuesta satisfactoria al tratamiento anticoagulante y trombolítico. En concordancia con lo sugerido por estudios ${ }^{16,17}$ y meta-análisis ${ }^{14}$, esta técnica quirúrgica resultó en mejoría significativa de los parámetros hemodinámicos, CF y sintomatología del paciente.

A los pacientes que presentan TEP significativo, valores relativamente bajos de RVP y síntomas moderados también se les ofrecería PTE para mejorar tolerancia al ejercicio y así prevenir la HTP por arteriopatía de vaso pequeño ${ }^{16}$. Estudios recientes han propuesto la angioplastía pulmonar con balón (BPA) como tratamiento para pa- cientes que presentan contraindicación a la $\mathrm{PTE}^{18}$. Esta técnica posibilita la intervención de segmentos más distales de la circulación pulmonar, muchas veces no abordables por PTE. Sepúlveda y cols., comunican una serie de ocho pacientes considerados inoperables que fueron tratados con angioplastía guiada por Optical Frequency Domain Imaging, como alternativa segura y efectiva, con excelentes resultados hemodinámicos inmediatos y clínicos a corto plazo ${ }^{19}$.

El manejo postoperatorio implica anticoagulación precoz, considerando el estado de la volemia, precarga, gasto cardíaco y consumo de oxígeno.

La mayoría de los pacientes muestra disminución significativa en la PMAP y la RVP, con excelente sobrevida a largo plazo. Es importante destacar que la sobrevida del paciente a 5 años mejora aún en casos en que el paciente quede con HTP residual tras la cirugía ${ }^{16}$. El tratamiento médico mejora parámetros hemodinámicos y la capacidad al ejercicio en los pacientes con contraindicación a la cirugía. Sin embargo, no existen estudios randomizados ni consenso sobre su real utilidad. En algunos casos está indicado como puente a la cirugía o en HTP residual postoperatoria. No es superior al tratamiento quirúrgico ${ }^{1,20}$. En conclusión, la CTPH es una causa de HTP posible de curar en algunos pacientes mediante la PTE. El diagnóstico precoz de esta condición patológica de mal pronóstico es fundamental para poder derivar a estos pacientes a centros de alta complejidad y con experiencia, mejorando así los resultados clínicos y la sobrevida.

\section{Agradecimientos:}

El Departamento Cardiovascular del Hospital Clínico de la Universidad de Chile y los autores del manuscrito agradecen la colaboración en calidad de Primer Cirujano y Proctor al Dr. Michael M. Madani, División of Cardiovascular and Thoracic Surgery, University of California, San Diego, Medical Center, San Diego, California, USA. 


\section{Referencias}

1- ALI JM, HARDMAN G, PAGE A, JENKINS DP. Chronic thromboembolic pulmonary hypertension: an underdiagnosed entity? Hosp Pract. 2012; 40: 71-79.

2- SISTA, AKHILESH K., AND FREDERIKUS A. KLOK. Late Outcomes of Pulmonary Embolism: The Post-PE Syndrome. Thromb Res 2018; 164 (April): 157-62.

3- ENDE-VERHAAR, YVONNE M., SUZANNE C. CANNEGIETER, ANTON VONK NOORDEGRAAF, MARION DELCROIX, PIOTR PRUSZCZYK et al. Incidence of Chronic Thromboembolic Pulmonary Hypertension after Acute Pulmonary Embolism: A Contemporary View of the Published Literature. Eur Respir J 2017; 49 (2).

4- JENKINS D, MADANI M, FADEL E, D'ARMINI AM, MAYER E. Pulmonary endarterectomy in the management of chronic thromboembolic pulmonary hypertension. Eur Respir Rev. 2017; 26 (143).

5- GALIÈ, NAZZARENO, MARIUS M. HOEPER, MARC HUMBERT, ADAM TORBICKI, JEAN-LUC VACHIERY et al. Guidelines for the Diagnosis and Treatment of Pulmonary Hypertension: The Task Force for the Diagnosis and Treatment of Pulmonary Hypertension of the European Society of Cardiology (ESC) and the European Respiratory Society (ERS), Endorsed by the International Society of Heart and Lung Transplantation (ISHLT). Eur Heart J 2009; 30 (20): 2493-2537.

6- WIETESKA M, BIEDERMAN A, KURZYNA M, DYK W, BURAKOWSKI J, WAWRZYŃSKA L, et al. Outcome of Medically Versus Surgically Treated Patients With Chronic Thromboembolic Pulmonary Hypertension. Clin Appl Thromb Hemost. 2016; 22: 92-99.

7- MATTHEWS DT, HEMNES AR. Current concepts in the pathogenesis of chronic thromboembolic pulmonary hypertension. Pulm Circ. 2016; 6: 145-154.

8- LANG IM, PESAVENTO R, BONDERMAN D, YUAN JX-J. Risk factors and basic mechanisms of chronic thromboembolic pulmonary hypertension: a current understanding. Eur Respir J. 2013; 41: 462-468.

9- LANG, IRENE M., PETER DORFMÜLLER, AND ANTON VONK NOORDEGRAAF. The Pathobiology of Chronic Thromboembolic Pulmonary Hypertension. Annals of the American Thoracic Society. 2016; 13 Suppl 3 (July): S215-21.

10- MEMON HA, LIN CH, GUHA A. Chronic Thromboembolic Pulmonary Hypertension: Pearls and Pitfalls of Diagnosis. Me- thodist Debakey Cardiovasc J. 2016;12: 199-204.

11- FEDULLO P, KERR KM, KIM NH, Auger WR. Chronic thromboembolic pulmonary hypertension. Am J Respir Crit Care Med. 2011;183: 1605-1613.

12- MADANI MM, JAMIESON SW. Technical advances of pulmonary endarterectomy for chronic thromboembolic pulmonary hypertension. Semin Thorac Cardiovasc Surg. 2006;18: 243-249.

13- MADANI MM, AUGER WR, PRETORIUS V, SAKAKIBARA $\mathrm{N}, \mathrm{KERR} \mathrm{KM}, \mathrm{KIM} \mathrm{NH}$, et al. Pulmonary endarterectomy: recent changes in a single institution's experience of more than 2,700 patients. Ann Thorac Surg. 2012;94: 97-103; discussion 103.

14- RAHNAVARDI M, YAN TD, CAO C, VALLELY MP, BANNON PG, WILSON MK. Pulmonary thromboendarterectomy for chronic thromboembolic pulmonary hypertension: a systematic review. Ann Thorac Cardiovasc Surg. 2011;17: 435-445.

15- ZALAQUETT RICARDO, BECKER PEDRO, IRARRÁZABAL MANUEL, MORÁN SERGIO, MATURANA GUSTAVO et al. Tromboendarterectomía pulmonar. Tratamiento quirúrgico del tromboembolismo pulmonar crónico bajo paro circulatorio con hipotermia profunda en un caso. Rev Méd Chile $1996 ; 124: 847-854$

16- FREED DH, THOMSON BM, BERMAN M, TSUI SSL, DUNNING J, SHEARES KK, et al. Survival after pulmonary thromboendarterectomy: effect of residual pulmonary hypertension. J Thorac Cardiovasc Surg. 2011;141: 383-387.

17- PORRES-AGUILAR M, ANAYA-AYALA JE, PORRES MUÑOZ M, BRACAMONTES F. Tromboendarterectomía pulmonar en pacientes con hipertensión arterial pulmonar tromboembólica crónica. Cir Ciruj 2007; 75: 131-8.

18- AHN CM, HIROMI M. Chronic Thromboembolic Pulmonary Hypertension: Endovascular Treatment. Korean Circ J. 2019;49: 214-222.

19- SEPÚLVEDA PABLO, ORTEGA JUAN, ARMIJO GERMÁN, TORRES JOSÉ, RAMÍREZ PABLO, BACKHOUSE CHRISTIAN et al. Angioplastía con balón para el tratamiento de la hipertensión arterial pulmonar por enfermedad tromboembólica crónica. Resultados hemodinámicos y clínicos inmediatos. Rev. méd. Chile 2019; 147: 426-436.

20- PEPKE-ZABA J, GHOFRANI H-A, HOEPER MM. Medical management of chronic thromboembolic pulmonary hypertension. Eur Respir Rev. 2017;26. 